\title{
EXTRACTIVISMO Y TERRITORIO: EL ORDENAMIENTO TERRITORIAL COMO HERRAMIENTA PARA LA GESTIÓN DE CONFLICTOS SOCIALES
}

\author{
EXTRACTIVISM AND TERRITORY: LAND-USE \\ PLANNING AS A TOOL FOR MANAGING \\ SOCIAL CONFLICTS
}

\author{
FLAVIO VILA \\ Universidad de Lima \\ orcid.org/0000-0002-6056-6335
}

En el Perú la extracción de recursos naturales en alta intensidad ocasiona la mayoría de sus conflictos sociales. Esta industria, basada en el "crecimiento infinito" del sistema civilizado capitalista, colisiona con la cosmovisión de los pueblos indígenas al sembrar un campo insostenible de conflicto latente. Consecuencias territoriales de impacto negativo añaden inestabilidad, lo que incrementa la percepción de injusticia y el escalamiento del conflicto. El ser humano interactúa a diario con su territorio, pero su planificación está ausente en entornos de extractivismo. El presente artículo tiene como objetivo evidenciar la urgencia de ordenar estos territorios a partir de la identificación de una relación entre la gestión de conflictos sociales y el ordenamiento territorial, enmarcado en el decrecimiento como transición a un pluriverso ontoepistémico. Es una investigación aplicada, donde se expone la problemática en entornos de extractivismo y luego se propone un modelo de resolución práctica para esta.

territorio, extractivismo, conflictos sociales, crecimiento, decrecimiento
Recibido: 1 de julio del 2020

Aprobado: 6 de octubre del 2020

doi: https://doi.org/10.26439/limaq2021.n008.5553

In Peru, the extraction of natural resources in high intensity causes most of its social conflicts. This industry, based on the 'infinite growth' of the capitalist civilizing system, collides with the worldview of Indigenous Peoples by sowing an unsustainable field of latent conflict. Territorial consequences of negative impact add instability, which increases the perception of injustice and the escalation of the conflict. Human beings interact with their territory daily, but its planning is absent in extractive environments. This article aims to portray the urgency of ordering these territories from the identification of a relationship between the social conflicts management and the territorial ordering, framed in degrowth as a transition to a pluriversal civilizing system. It is applied research where the problem in extractive environments is exposed and then a practical resolution model is proposed for it.

territory, extractivism, social conflicts, growth, degrowth 


\section{INTRODUCCIÓN}

Actualmente, el Perú apuesta por el extractivismo de alta intensidad; sin embargo, este ocasiona el $53 \%$ de sus conflictos sociales ${ }^{1}$ (Defensoría del Pueblo, 2020). Las actividades extractivas suelen suceder en entornos donde se despojan derechos territoriales. El Estado, desde su modelo neoliberal capitalista, promueve la extracción de recursos naturales, lo que usualmente resulta en la degradación del suelo para actividades agropecuarias. En contraste, los pueblos indígenas, habitantes ancestrales de estos territorios, se oponen a esta extrahección defendiendo su relacionalidad con la naturaleza y formaciones ontoepistémicas. Estas son el conjunto de relaciones sociales, lenguajes, entramados comunitarios, marcos valorativos y estructuras psicosociales que nacen alrededor de maneras distintas de percibir el mundo y el conocimiento creado en este proceso subjetivo/objetivo (Gómez Liendo y Olmedo Neri, comunicación personal, 14 de mayo del 2020). Por esta razón, este artículo propone que la gestión de conflictos sociales debe plantearse desde el ordenamiento territorial, pues es esta disputa por territorios la que principalmente los genera.

Los tres términos en los que se basa la investigación para la defensa de esta postura son extractivismo, conflicto social y territorio, definidos a continuación. Primero, extractivismo, tomando la definición de Gudynas (2013), es un tipo particular de extracción de recursos naturales, en gran volumen e intensidad, mayormente destinados a la exportación como materias primas sin procesar o con poco procesamiento. Incluye también las fases previas de exploración y las posteriores de cierre hasta el abandono de la apropiación (p. 15). Segundo, para conflicto social se tomó la definición de la Defensoría del Pueblo (2020), ya que esta institución será referenciada más adelante. Según esta, un conflicto social es "un proceso complejo en el cual sectores de la sociedad, el Estado y empresas perciben que sus objetivos, intereses, valores o necesidades son contradictorios y esa contradicción puede derivar en violencia" (p. 3) ${ }^{2}$. El último es territorio, que se definió desde una postura propia, basada en experiencias profesionales y académicas, como una delimitación espacio-temporal con noción de propiedad, producto de todas las relaciones socioculturales, económicas y ecosistémicas que suceden en dicha delimitación. Para explicar esta definición, se debe comenzar asumiendo que el espacio-tiempo es infinito y único, pero son las nociones de propiedad las que lo delimitan o fragmentan en lo que llamamos territorio. Estas nociones pueden ser jurisdiccionales (distrito, provincia, región, etcétera) o culturales (barrios, territorios indígenas, etcétera), y se definen según las relaciones mencionadas previamente. Esto implica que diferentes grupos de la población pueden tener sentidos de propiedad distintos sobre el mismo espacio-tiempo

1 Cálculo basado en los conflictos sociales causados por minería e hidrocarburos

2 La Defensoría del Pueblo también agrega que el conflicto es inherente a la vida, al ser una característica natural e inevitable de la existencia humana y de la interacción social. Además, señala que conflicto y violencia no son lo mismo; la violencia es la manifestación destructiva del conflicto social. 
y esto puede derivar en un conflicto social; por ejemplo, un territorio indígena que se encuentra dividido por un límite provincial donde distintos intereses concernientes a su uso resultan contradictorios.

Esta es una investigación aplicada en la que se analizó una problemática a través de un caso de estudio mediante la aplicación de investigaciones y teorías; luego se propuso un modelo para su resolución práctica. Está compuesta por cuatro secciones: primero, se explicó el conflicto latente en entornos de extractivismo donde la lógica del crecimiento infinito capitalista es impuesta sobre las economías de subsistencia de los territorios indígenas; esto genera un campo inestable de opresión cultural. Luego, se analizó cómo el extractivismo transforma el territorio en el caso de Cerro de Pasco en tres momentos específicos de su historia; a partir de este caso, se añadieron investigaciones y teorías con las cuales se demostró que esta transformación incrementa el descontento de la población y escala hacia el conflicto social. Posteriormente, se introdujo el concepto de decrecimiento, el cual es un discurso de transición hacia un modelo alterno al crecimiento capitalista, y se definieron cuáles deberían ser los límites de dicho crecimiento. Por último, en el apartado de conclusiones y recomendaciones, se planteó al ordenamiento territorial, enmarcado en el decrecimiento, como una herramienta para la gestión de conflictos sociales con el fin de remediar las problemáticas descritas anteriormente.

\section{COLISIÓN DE COSMOVISIONES: UN CONFLICTO LATENTE}

Es imposible hablar de extractivismo y sus consecuencias territoriales sin comprender primero el sistema civilizatorio en el cual se enmarca. La lógica del capital, definida por sus formas específicas de apropiación y explotación de los bienes naturales, se encuentra sumamente relacionada con la conflictividad social actual. Las formas contemporáneas de control del capital sobre la naturaleza ${ }^{3}$ están basadas en el crecimiento infinito, es decir, en la búsqueda perpetua de la máxima ganancia, que es reinvertida nuevamente en el sistema para ser incrementada. Es un bucle de retroalimentación positivo en el que la acumulación es cada vez más amplia, los recursos naturales son depredados y la economía no crece per se, sino que es la búsqueda de la máxima ganancia el motor de dicho crecimiento (Pineda, 2018, p. 127-128).

Contextualizando esto en el entorno extractivo latinoamericano, estos mecanismos de mercantificación del territorio desencadenan una colisión de cosmovisiones ${ }^{4}$ donde el modelo desarrollista importado es impuesto ${ }^{5}$

3 Estos son los mecanismos de despliegue de dominio que aplica el modelo capitalista sobre el territorio global. Se pueden reconocer cinco formas o tipos de megaproyectos: procesos extractivos, procesos agroindustriales, procesos de hiperurbanización, megaestructura energética y comunicacional, y procesos de biomercantilización (Pineda, 2018, pp. 135-143).

4 Véase la "tensión de territorialidades" en Porto Gonçalves (2001) y la "percepción de la realidad" en Lorenzo Cadarso (2001).

5 Históricamente, el Occidente se ha autoproclamado el derecho de ser "el mundo" sometiendo a cosmovisiones alternas a un estado de inexistencia (Law, 2011, p. 1). 
sobre el holismo comunitario presente desde tiempos ancestrales prehispánicos. En 1532, los españoles fuerzan el colonialismo sobre los pueblos indígenas peruanos empezando con transformaciones en el territorio como las reducciones indígenas ${ }^{6}$ y la fundación de nuevas ciudades. Con la independencia en 1821 se deja de lado la dominación colonial reemplazándola por el neocolonialismo ${ }^{7}$, nueva dominación que se ha mantenido a lo largo de los siglos mediante megaproyectos e imperialismo cultural sobre los pueblos originarios. Es en esta imposición histórica y constante donde yace el conflicto latente que existe en entornos de extractivismo latinoamericano. Las sociedades rurales campesinas se caracterizan por una actividad económica de autoabastecimiento basada en los recursos locales y limitada por los ciclos naturales; por otro lado, las sociedades urbano-industriales fundan su economía en la extracción de recursos y producción a gran escala para la mercantilización del producto (López García y López López, 2003, p. 54).

La lógica capitalista trabaja desvinculando a la comunidad de su territorio al aplicar estrategias como la privatización de tierras, la expulsión forzada de poblaciones campesinas, la conversión de derechos comunales a privados, la supresión de acceso a bienes comunales, la mercantilización de la fuerza de trabajo y la supresión de formas alternativas de producción (Harvey, 2003, p. 116). La ausencia de un mediador público ${ }^{8}$ respalda estos procesos facilitando la desarticulación con la naturaleza. Las relaciones comunales y sociales no mercantilizadas se vuelven un obstáculo para la expansión del capital, ya que muchas veces oponen resistencia al proceso de desposesión (Pineda, 2018, p. 135). Esta separación entre hombres y naturaleza es uno de los pilares de nuestro sistema moderno, pues la producción se basa en la explotación de recursos naturales ${ }^{9}$. El racionalismo moderno imagina el mundo como una superficie inanimada para ocupar, mientras que muchas culturas lo consideran un espacio vivo para habitar (Escobar, 2016, p. 105). Sumado a esto, y aplicando la teoría marxista de la alienación (Sossa Rojas, 2010, pp. 39-45), esta comunidad desvinculada de su territorio tradicional se convierte en la fuerza de trabajo de la industria extractiva. Esta transformación repentina afecta sobre todo a las mujeres reconfigurando los roles de género

6 Unidad territorial creada para agrupar a la población andina dispersa en centros urbanos que, según las autoridades españolas, debía ser incorporada a la vida en urbanidad y buenas costumbres (González Díaz, 2018, párr. 2).

7 El colonialismo tiene como objetivo la creación de colonias con el fin de explotar los recursos de otros territorios aplicando la dominación militar en el proceso. En el neocolonialismo esta dominación se vuelve económica, ya que la colonia independiente continúa siendo dependiente del capital extranjero.

8 Las leyes peruanas no incorporan un reconocimiento a la interculturalidad como fuente de derechos (Correa Aste, 2011, p. 14).

9 Marx identifica dos grandes opresiones del capitalismo, con la naturaleza y con la fuerza laboral, llamándolas contradicciones. La descrita es la "contradicción capital-naturaleza", donde la explotación de recursos naturales es parte de nuestro sistema civilizatorio; sin opresión de la naturaleza no hay mercado. 
de la sociedad rural. Los varones son captados por las empresas extractivas ${ }^{10}$ dejando a las mujeres recluidas en el ámbito doméstico y encargándose de actividades agropecuarias que eran previamente compartidas. Esta sobrecarga de tareas dificulta su acceso a la educación o a la participación en espacios de decisión (Delbene-Lezama, 2015, pp. 7-8), problemáticas que han sido identificadas por las corrientes ecofeministas.

Finalmente, esta alienación también es territorial porque la actividad extractiva produce cambios físicos observables, como nuevas urbanizaciones, por ejemplo. Sin embargo, el territorio no solo es una delimitación espacio-temporal, sino que es el producto de todas las relaciones socioculturales, económicas y ecosistémicas que suceden en esa delimitación. Dicho esto, las consecuencias ontológicas son igual de importantes, pues el mundo se percibe de distintas maneras. En la cosmovisión andina, en particular, este es un sistema holístico donde los seres vivos (humanos, animales, plantas) y no vivos (piedras, ríos, cerros) son parte de un mismo organismo viviente (Quispe, 2013, párrs. 1-5). La aparición de un "tajo abierto"11 donde antes se ubicaba un $a p u^{12}$ es un conflicto de carácter psicogeográfico que nace desde la concepción cultural de un espacio; diversas cosmovisiones indígenas sostienen debates políticos con seres no humanos, ya que ciertos animales o accidentes geográficos tienen voluntad propia, política y son sujetos morales ${ }^{13}$ (Gudynas, 2014, p. 7).

Entonces, en medio de esta problemática estructurante, ¿ cómo se pretenden gestionar conflictos sociales con un grupo poblacional al que se le ha oprimido durante quinientos años de manera sociocultural y territorial? Evidentemente, para lograr esto, se debe abandonar el sistema civilizatorio descrito anteriormente y repensar lo que se entiende por "desarrollo". La misión del "crecimiento de regiones subdesarrolladas", propuesta por Harry Truman en $1949^{14}$, demuestra la creencia en un mundo único y perfecto a emular (Escobar, 2016, p. 104), basado en la lógica del crecimiento infinito que ha concebido el oxímoron del "desarrollo sostenible" para justificar sus lógicas depredadoras sin juicios morales (Pineda, 2018; Gómez-Baggethun, 2019; Olmedo Neri, 2019). Sin embargo, las palabras crecimiento y desarrollo no son sinónimas. Por ejemplo, pensar que crecimiento económico y desarrollo

10 Este grupo de la población pierde sus valores "humanos" y es mercantilizado convirtiéndose en un medio o herramienta para lograr la máxima ganancia. Esta opresión es la "contradicción capital-trabajo", la segunda contradicción que se mencionó anteriormente.

11 Método de minado superficial.

12 Significa "señor" en quechua; se refiere a montañas divinas consideradas ancestros por las comunidades indígenas.

13 Las ecologías políticas debaten sobre la inclusión de la naturaleza como sujeto jurídico en las constituciones latinoamericanas concibiendo una política con participantes humanos y no humanos.

14 Véase el cuarto punto del llamado "Discurso de los cuatro puntos" de Harry Truman en enero de 1949. 
económico significan lo mismo es caer en el error. Crecer significa incrementar un tamaño por acumulación, mientras que desarrollar significa mejorar para lograr la realización de un potencial. El crecer tiene implicancias cuantitativas al hacer algo cuantitativamente más grande; el desarrollar, por otro lado, es cualitativo al hacer algo cualitativamente mejor (Meadows et al., 1994, p. 28). Esta distinción nos indica, en palabras de Gudynas (2009), que es necesario romper con la idea del crecimiento económico como motor del desarrollo y, en cambio, colocar el acento en la calidad de vida (p. 16).

Es en esta transición civilizatoria donde aparecen nuevos discursos y paradigmas que implican

el desarrollo de tecnologías (y procesos) que permitieran explotar los recursos naturales con el mínimo impacto ambiental, reformas sociales y económicas hacia la equidad, así como profundos cambios en el comportamiento de la población en relación con el uso de energía y el consumo de bienes y servicios. (Parra Romero y Cadena Díaz, 2010, p. 340)

Una transición donde el ordenamiento territorial es aplicable como una herramienta para la gestión de conflictos sociales.

LAS CONSECUENCIAS TERRITORIALES DEL EXTRACTIVISMO

El conflicto latente de cosmovisiones descrito en la sección anterior es el equivalente al tablero de juego en entornos extractivos: un campo inestable e insostenible con las condiciones sembradas para el escalamiento de conflictos sociales. Además de esto, se tienen las consecuencias territoriales producidas por el extractivismo que, al añadir inestabilidad, llevan a situaciones donde un infortunio puede activar el conflicto latente mencionado ${ }^{15}$. Para fines de esta investigación, se tomó como caso la ciudad de Cerro de Pasco en tres momentos de su historia. Luego, para acotar la problemática, a este análisis se le sumaron otras situaciones socioeconómicas y ambientales de impacto negativo. Estas son (1) la incorrecta inversión de los fondos públicos; (2) la degradación del medio de vida agropecuario; (3) la frustración de expectativas definida como "la divergencia entre lo que un grupo social espera y cree merecer recibir y lo que realmente obtiene" (Lorenzo Cadarso, 2001, p. 244); y (4) la dependencia económica extractiva.

Las actividades extractivas están sometidas a presiones migratorias enormes que catalizan procesos no planificados de urbanización en los centros poblados (Thodes Miranda, 2016, p. 205). Esto da lugar a múltiples cambios territoriales como expansiones urbanas, creación de enclaves ${ }^{16}$, reasentamientos involuntarios, creación de nuevos centros poblados, declive de ciudades,

15 En el Perú, según la Defensoría del Pueblo (2020), un conflicto puede tener tres estados: latente, activo y resuelto.

16 Paisajes urbanos fragmentados provocados por el desarrollo de zonas residenciales, centros comerciales y áreas de servicios destinados a la élite de las empresas mineras, pero incapaces de atender las necesidades de todos los habitantes (Angotti, 2013; Thodes Miranda, 2016). 
etcétera (Gonzales Gavilano, 2017, p. 510). Sin embargo, el vínculo entre la actividad extractiva y el urbanismo es un campo poco explorado (De Echave et al., 2007; Gonzales Gavilano, 2017), así que con flujos migratorios masivos descontrolados la expansión física es caótica. Probablemente, el caso más emblemático de la situación descrita es la ciudad de Cerro de Pasco, capital de la región Pasco, donde décadas de expansión urbana descontrolada y explotación minera han ocasionado que la actividad extractiva suceda en plena ciudad (véase la figura 1). Es una situación virtualmente imposible de revertir, ya que, paradójicamente, la ampliación del "tajo abierto" se ha convertido también en su bonanza económica. El cierre de la mina significaría la pobreza de toda la región, argumento usado por los distintos propietarios durante la última mitad del siglo para justificar la expansión del "tajo". Sin embargo, esta resulta en la depredación de la trama urbana y el patrimonio histórico (véase la figura 2); la ciudad debe elegir constantemente entre perder su pasado o su futuro (Vittor, 2007, párrs. 1-4).

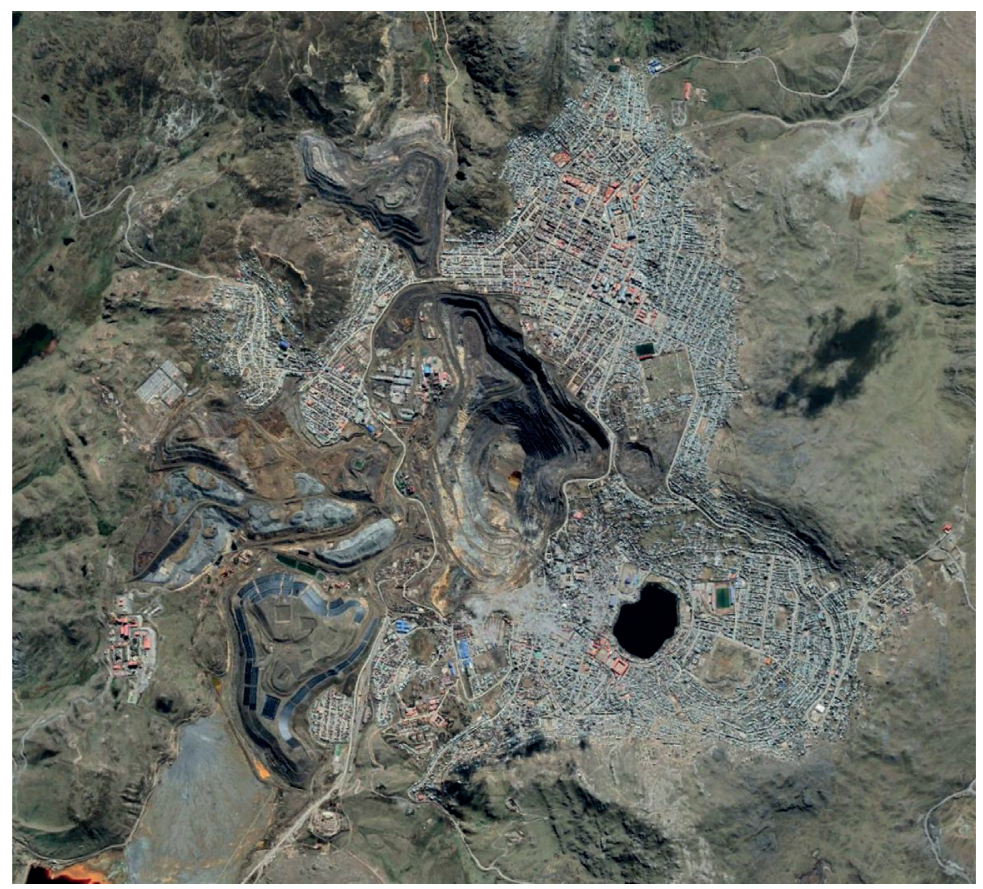

Figura 1.

Foto satelital de

Cerro de Pasco: la minería sucede al centro de la ciudad

Fuente: Google Earth (s. f.) 
Figura 2 .

El "tajo abierto" ha ido devorando el casco urbano

Fotografía: Van Houtryve (2015)

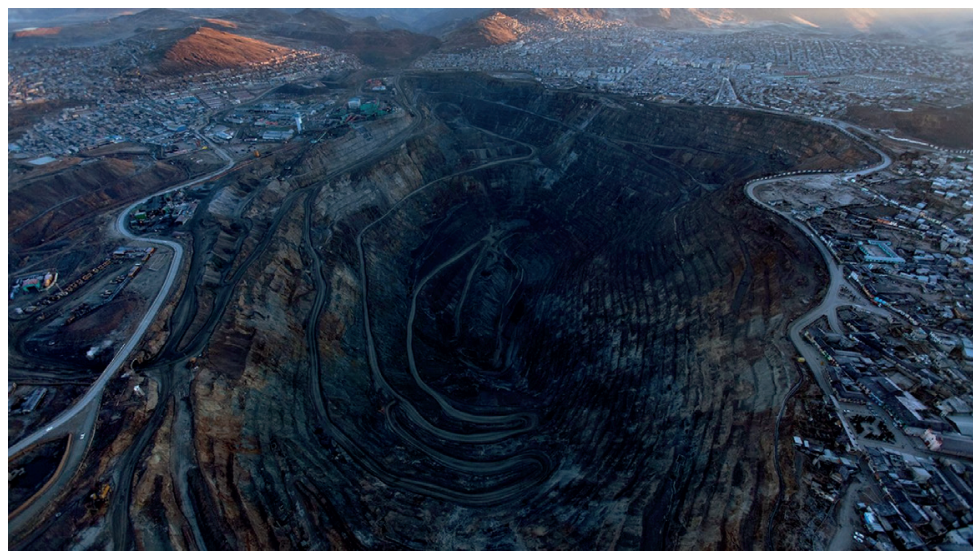

No obstante, la situación podría ser distinta para Cerro de Pasco si se hubieran tomado otras decisiones durante el siglo pasado. Fueron tres momentos de su historia contemporánea en los que hubo conflictos por reasentamiento, siempre inmediatamente después de momentos icónicos para la expansión minera:

1. En 1904, después de la construcción del ferrocarril Callao-Cerro de Pasco que consolidó el enclave minero y llevó al municipio a presentar reclamos judiciales para el cierre de la mina. En respuesta, la Cerro de Pasco Copper Corporation presentó al gobierno un proyecto para el traslado de la población que nunca se concretó.

2. En 1956, después de iniciarse la explotación a "tajo abierto" que transformaría permanentemente la vida urbana. Esta vez se propusieron dos opciones de reasentamiento: Villa Pasco, $20 \mathrm{~km}$ al sur de la ciudad antigua, y San Juan Pampa, 1,5 km al norte. La Cerro de Pasco Copper Corporation, que debía asumir los gastos, propuso San Juan Pampa por ser más barata; y en 1971, el dictador Velasco Alvarado, que tres años después la estatizaría convirtiéndola en la Empresa Minera del Centro del Perú (Centromin), respaldó la propuesta mediante un decreto ley. Actualmente, San Juan Pampa, debido a la expansión urbana y minera, ya es parte del casco urbano de la ciudad y se ubica a cuadras del "tajo abierto", mientras que Villa Pasco continúa siendo un área apropiada para una posible expansión urbana.

3. En el 2007, cuando Volcan Compañía Minera, actual propietaria de la mina, presentó un nuevo plan de expansión que afectaría espacios públicos, viviendas e inmuebles, sobre todo la plaza Chaupimarca y la iglesia matriz, que se encuentran en la memoria histórica de la ciudad. Desde 1956, la ciudad antigua, que ya casi no existe, había sido depredada y, por esta razón, este nuevo plan causó rechazo entre la población. No se ha llevado a cabo aún y será muy complicado hacerlo sin una visión concertada de desarrollo urbano (Vittor, 2007, párrs. 6-23). 
Esta migración, que cataliza la expansión física de un centro poblado, está relacionada con la explotación extractiva y es una reacción natural de las comunidades ante la promesa de desarrollo y riqueza. La tabla 1 muestra la población censada de la ciudad de Cerro de Pasco comparada con la de Lima Metropolitana, a la cual se le considera el epítome del desborde popular en el Perú. Sin embargo, se puede observar que el incremento intercensal de Cerro de Pasco superó al de Lima en dos censos, 1972 y 1981, probablemente relacionados con la continua ampliación del "tajo abierto". Con migraciones que triplican una población en veinte años, de 21363 en 1961 a 66373 en 1981, y sin una visión de planificación urbana o territorial a largo plazo, no parece muy descabellado esperar un resultado como el que actualmente enfrentan los cerreños.

\begin{tabular}{|c|c|c|c|}
\hline & & Cerro de Pasco & Lima Metropolitana \\
\hline 1940 & Población & 17882 & 645172 \\
\hline \multirow[t]{3}{*}{1961} & Población & 21363 & 1845910 \\
\hline & Valor absoluto & 3481 & 1200738 \\
\hline & $\begin{array}{l}\text { Incremento intercensal } \\
\text { (porcentaje) }\end{array}$ & $19,5 \%$ & $186,1 \%$ \\
\hline \multirow[t]{3}{*}{1972} & Población & 46907 & 3302523 \\
\hline & Valor absoluto & 25544 & 1456613 \\
\hline & $\begin{array}{l}\text { Incremento intercensal } \\
\text { (porcentaje) }\end{array}$ & $119,6 \%$ & $78,9 \%$ \\
\hline \multirow[t]{3}{*}{1981} & Población & 66373 & 4573227 \\
\hline & Valor absoluto & 19466 & 1270704 \\
\hline & $\begin{array}{l}\text { Incremento intercensal } \\
\text { (porcentaje) }\end{array}$ & $41,5 \%$ & $38,5 \%$ \\
\hline \multirow[t]{3}{*}{1993} & Población & 62749 & 6321173 \\
\hline & Valor absoluto & -3624 & 1747946 \\
\hline & $\begin{array}{l}\text { Incremento intercensal } \\
\text { (porcentaje) }\end{array}$ & $-5,5 \%$ & $38,2 \%$ \\
\hline \multirow[t]{3}{*}{2007} & Población & 66860 & 8472935 \\
\hline & Valor absoluto & 4111 & 2151762 \\
\hline & $\begin{array}{l}\text { Incremento intercensal } \\
\text { (porcentaje) }\end{array}$ & $6,6 \%$ & $34,0 \%$ \\
\hline \multirow[t]{3}{*}{2017} & Población & 58899 & 9562280 \\
\hline & Valor absoluto & -7961 & 1090188 \\
\hline & $\begin{array}{l}\text { Incremento intercensal } \\
\text { (porcentaje) }\end{array}$ & $-11,9 \%$ & $12,9 \%$ \\
\hline
\end{tabular}

\section{Tabla 1}

Población censada e incremento intercensal de Cerro de Pasco y Lima Metropolitana

Elaboración propia sobre la base de Instituto Nacional de Estadística e Informática (1995, 2008, 2018) 
Como se mencionó a inicios de esta sección, a esta alienación territorial se le suman otras situaciones socioeconómicas y ambientales de impacto negativo que se retroalimentan en la conflictividad latente, acumulando inestabilidad y escalando el conflicto social. En primer lugar, la importancia de un instrumento de planificación no solo recae en ordenar la expansión física caótica, sino también en guiar la inversión correcta de los fondos públicos y privados. La población - originaria y migrante- de un centro poblado en área de influencia extractiva se traduce en una cantidad de necesidades por ser abastecidas. Estas podrían suministrarse invirtiendo el canon, las regalías y las obras por impuestos en proyectos sociales y de infraestructura. El Estado peruano cuenta con la Ley del Canon ${ }^{17}$, la cual dicta que "los recursos que los gobiernos regionales y gobiernos locales reciban por concepto de canon serán utilizados para [...] obras de infraestructura. También podrán ser [...] destinados a proyectos de vivienda" (Ley 30848, 2018, artículo 2). Sin embargo, su inadecuada gestión no suple la vivienda, los equipamientos, la infraestructura y el espacio público que esta nueva población necesita.

Esta "inversión sin visión" tiene como consecuencia que el 32 \% del canon se ejecute sin proyecto de inversión, plan u objetivo; simplemente se gasta o desaparece por corrupción (Beteta, 2019, párr. 5). La Sociedad Nacional de Minería, Petróleo y Energía (SNMPE) cuestiona la forma en que se destinan estos fondos, que son gastados en "elefantes blancos" como piscinas en distritos sin agua, estadios con capacidad mayor a toda la población o monumentos diversos (véase la figura 3). Al no definir cuáles son las verdaderas necesidades que existen en las jurisdicciones, la mayoría de la población permanece en la pobreza (El Comercio, 2014, párrs. 4-5). Por ejemplo, en el año 2005, el $71,7 \%$ de la población de Cerro de Pasco era considerada pobre, según el método de necesidades básicas insatisfechas $(\mathrm{NBI})^{18}$ (Ministerio de Vivienda, Construcción y Saneamiento, 2008, p. 40). Además, sumando las constantes promesas privadas de desarrollo que nunca llegan a materializarse, los espacios habitados se marginalizan; el ser humano interactúa a diario con su medio construido y la percepción de desigualdad, sobre todo en un entorno donde se generan grandes recursos económicos, frustra continuamente las expectativas de la población local.

17 Publicada en el 2002 con la Ley 27506 y modificada en el 2018 con la Ley 30848.

18 Se considera pobre por NBI a aquella población que reside en hogares con al menos una de las siguientes necesidades básicas insatisfechas: vivienda precaria, vivienda hacinada, acceso a desagüe, niños menores de 12 años que no asisten al colegio y alta dependencia económica (Feres y Mancero, 2001). 

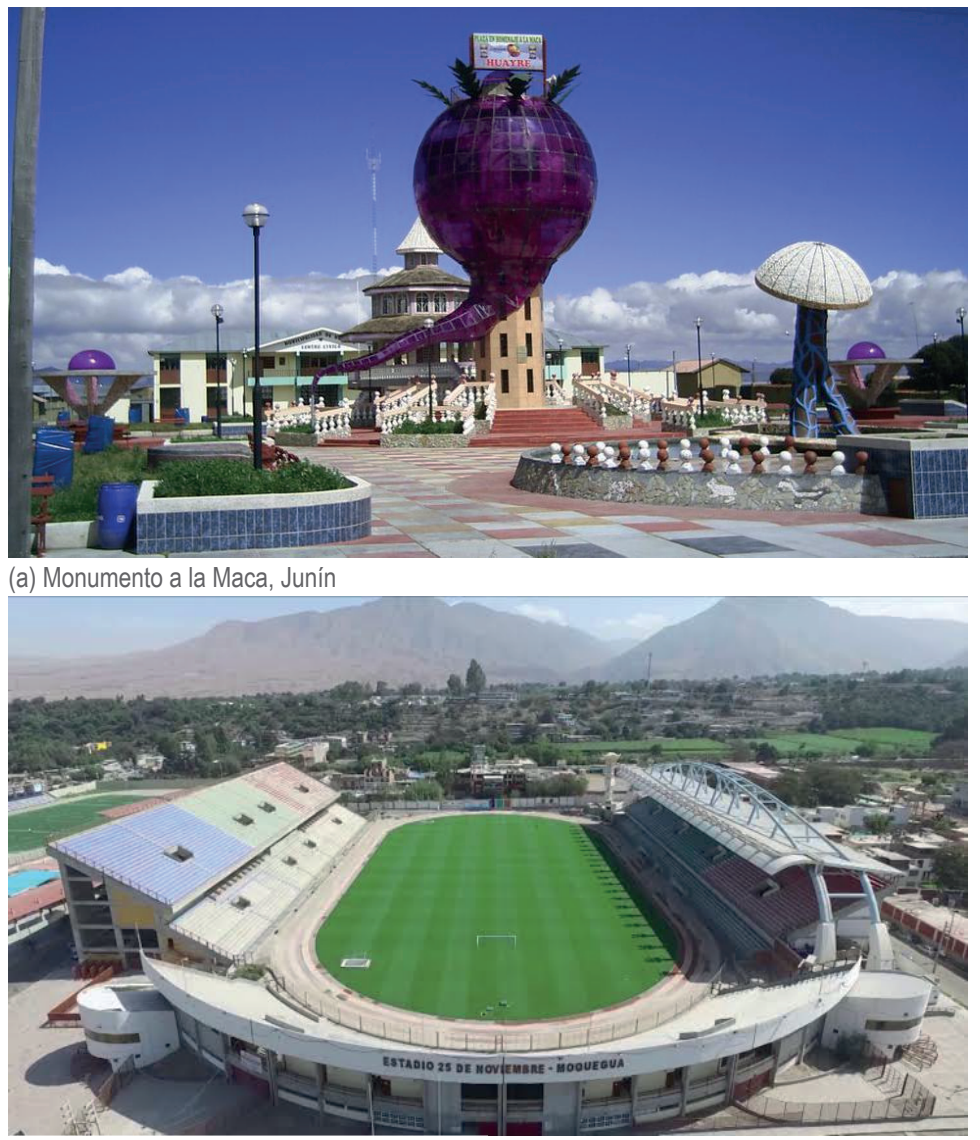

Figura 3. "Elefantes blancos": proyectos que no responden a las necesidades reales de la población

Fotografías:

(a) Perú21 (2013)

(b) Captura de video de Macrocontenidos (2017). (c) José Arturo Rodríguez (2012)

(b) Estadio 25 de Noviembre, Moquegua

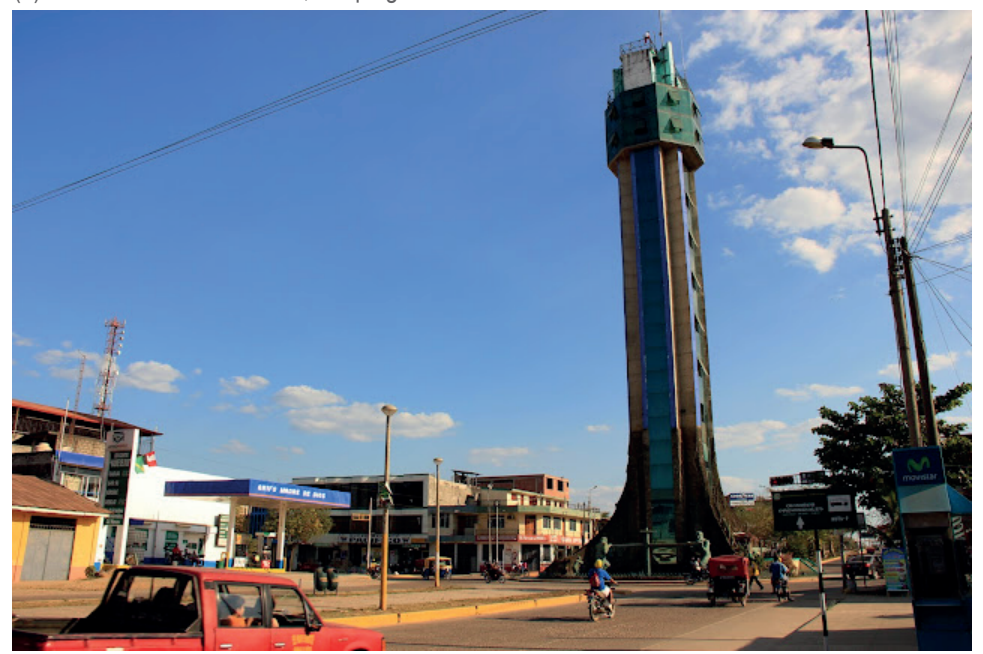

(c) Mirador de la Biodiversidad, Puerto Maldonado 
De hecho, investigaciones señalan que en el Perú la desigualdad incrementa la probabilidad de conflictos sociales en zonas de actividad minera, ya que en estos distritos las distribuciones de ingresos son más desiguales (Loayza y Rigolini, 2015; Castellares y Fouché, 2017). La tabla 2 muestra las principales razones de los conflictos mineros en el Perú, siendo la principal la contaminación y agricultura, porque la polución de los recursos hídricos y el suelo degrada el sustento agropecuario de las comunidades (De Echave et al., 2009, pp. 279-283). Además, se puede apreciar que muchas de estas razones pueden ser analizadas desde un enfoque territorial, sobre todo las relacionadas con agricultura y terrenos o áreas protegidas que físicamente ocupan un espacio en el territorio. El ordenamiento de estas áreas, sumado a los centros poblados, la actividad extractiva y las vías, en un instrumento de planificación tiene el fin de que dos actividades que actualmente se consideran mutuamente excluyentes, extractivismo y agricultura, puedan coexistir en un mismo entorno (De Echave et al., 2009, p. 313). En la misma línea, algunos acuerdos no cumplidos, específicamente los de infraestructura, se relacionan con los proyectos priorizados que necesitan estos centros poblados para abastecer sus nuevas necesidades. De esta manera, la planificación del territorio también transparenta la gestión de conflictos sociales convirtiendo al instrumento de ordenamiento en una herramienta de relaciones comunitarias.

\begin{tabular}{l|c|c}
\hline & $\begin{array}{c}\text { Proyectos en etapa de } \\
\text { explotación }\end{array}$ & Proyectos en etapa temprana \\
\hline $\begin{array}{l}\text { Contaminación ambiental/ } \\
\text { agricultura }\end{array}$ & $71 \%$ & $82 \%$ \\
Acuerdos no cumplidos & $23 \%$ & $11 \%$ \\
$\begin{array}{l}\text { Recursos económicos } \\
\text { adicionales }\end{array}$ & $18 \%$ & $6 \%$ \\
Beneficios laborales & $14 \%$ & $3 \%$ \\
Terrenosláreas protegidas & $3 \%$ & $11 \%$ \\
Minería ilegal & $6 \%$ & $7 \%$ \\
Otros & $3 \%$ & \\
\hline
\end{tabular}

La frustración de expectativas al ver que la distribución incorrecta de los recursos económicos no ha logrado reducir las brechas de pobreza y los territorios se degradan incrementa el riesgo de que un conflicto social en estado latente pase a activo. Se pueden identificar cuatro fases en el escalamiento de los conflictos sociales (Johnson, 1966, pp. 45-47) que son relacionables con las dinámicas territoriales explicadas previamente:

1. El desequilibrio sistemático (Johnson, 1966, p. 81) o frustración sistémica (Feierabend et al., 1969, pp. 499-500) de la sociedad al percibir problemas estructurales no resueltos en el sistema. Se 
vincula a la colisión de cosmovisiones donde el interés sobre un mismo territorio es distinto para los actores involucrados, lo que siembra un conflicto latente.

2. La ausencia de reformas por parte de los sectores privados y públicos. Se relaciona con las promesas de desarrollo incumplidas y la falta de planificación espacial para guiar las inversiones hacia las necesidades de las personas evitando la marginalización de los centros poblados. En este punto del escalamiento se agotan las vías institucionales; los individuos ven necesaria la creación de un espacio propio y empiezan a surgir los movimientos sociales ${ }^{19}$.

3. Pérdida de legitimidad del sector público al percibir que el Estado no ejerce presión sobre el privado para solucionar las problemáticas anteriores o, peor aún, pareciera aliarse con este. A esto se le suma la percepción de desigualdad donde los individuos observan como la brecha de pobreza incrementa en lugar de reducirse. Se conoce esto como privación relativa y es un resentimiento de la población al no conseguir lo que cree merecer (Gurr, 1970, p. 24), pues los pueblos indígenas tienen derecho de propiedad sobre los territorios que tradicionalmente han ocupado ${ }^{20}$.

4. Un acontecimiento que activa el conflicto como los mencionados en la tabla 2 u otros, como la expropiación de tierras en condiciones consideradas injustas, crecimiento de las tarifas de servicios básicos o de la aprobación de regulaciones que no respetan la interculturalidad (Domínguez, 2015, p. 144).

Finalmente, no es posible hablar de territorio y no mencionar la variable temporal, que es de suma importancia en los centros urbanos mineros. Esto es porque las actividades extractivas son de temporalidad finita, al estar sujetas a la riqueza de sus yacimientos y el agotamiento de estos (Gonzales Gavilano, 2017, p. 514). Esta dependencia del extractivismo crea comunidades monoindustriales no resilientes que, cuando llega el cierre o fin de la actividad, se sumen en la pobreza por el cese del canon y el desempleo. Se conoce esto como "la maldición de los recursos" y sostiene que, paradójicamente, los países cuyos territorios son ricos en recursos naturales suelen tener desempeños económicos pobres (Ross, 1999, pp. 299-300). A escala internacional, se han visto las consecuencias de esta dependencia durante la última década cuando la caída en el precio de los commodities impulsó a los gobiernos latinoamericanos a incrementar el número de proyectos extractivos. Sin embargo, no todos

19 Es importante mencionar que el término movimiento social se encuentra actualmente en crisis por las distintas sociedades presentes en nuestros centros poblados. Lo que antes se consideraba como urbanidad y ruralidad son ahora conceptos que se han difuminado, sobre todo culturalmente. Al no poder definir una sola sociedad en movimiento, el concepto de sociedades en movimiento de Raúl Zibechi empieza a tomar relevancia (Rota Verbum, 2018). de la Declaración de las Naciones Unidas sobre los Derechos de los Pueblos Indígenas. 
estuvieron preparados para la caída del precio de las materias primas, como fue el caso de Venezuela y su actual recesión (Svampa, 2019, p. 38).

Al mencionar una variable temporal, se está hablando de planificación y, por consiguiente, de territorio. Por esta razón, el discurso del espacio-tiempo, a pesar de parecer exclusivamente metafísico, tiene también carácter técnico. La Comisión Brundtland (1987) detallaba:

La presión sobre los recursos aumenta cuando la gente carece de otras alternativas. [...] Cada persona (debe) disponer de un medio de subsistencia duradero, especialmente los de familias pobres o que viven en regiones sometidas a tensiones ecológicas. (p. 77)

Los horizontes de planificación (corto, mediano y largo plazo) deben también incluir la vida futura de estos centros poblados al considerar invertir los fondos públicos y privados en la potencialización de actividades económicas independientes de la actividad extractiva (Kunanayagam et al., 2001; Gonzales Gavilano, 2017). La naturaleza intercultural del territorio peruano permite identificar y potenciar otras actividades económicas (Montero Peña y Salazar Pérez, 2012, p. 79) o grupos poblacionales como las mujeres indígenas que quedan relegadas por la opresión patriarcal del extractivismo (DelbeneLezama, 2015, p. 8).

\section{EL DECRECIMIENTO Y SUS LÍMITES AL CRECIMIENTO}

"El crecimiento (infinito) es uno de los propósitos más necios jamás inventados por cualquier cultura" (Meadows, como se citó en Raworth, 2017, p. 39), ya que el sistema civilizador capitalista se encuentra en crisis al no poder solucionar los problemas que ha generado con sus propios paradigmas (Rota Verbum, 2018). Es decir, en entornos de extractivismo no es posible resolver la depredación de recursos naturales con más extracción. En la existencia de este mundo único a emular, se extinguen otros mundos, singulares e irremplazables, con sus propias formaciones ontoepistémicas. Por esta razón, es perentorio descolonizar nuestra civilización hacia un pluriverso de configuraciones socionaturales (Escobar, 2016) donde se priorice la comunicabilidad entre una multiplicidad de mundos culturales con base en un entendimiento ecológico y político compartido (Escobar, 2012, p. 39). Precisamente son estos saberes ancestrales los que contienen diversas visiones para la transición a este pluriverso y que reciben el nombre de discursos para la transición (DT) ${ }^{21}$ (Escobar, 2016).

Uno de estos DT es el decrecimiento, que reclama la descolonización de la política actualmente acaparada por el economicismo que prioriza el crecimiento económico como objetivo social. En cambio, propone la creación

21 Los DT son visiones que parten de la noción de que la crisis socioecológica contemporánea es inseparable del modelo de vida capitalista. Dentro de estos se encuentran el decrecimiento, el posextractivismo, lo relacional, lo ecozoico, el buen vivir, el sumaq kawsay, las alternativas al desarrollo, etcétera (Escobar, 2016). 
de límites al crecimiento para controlar nuestra presión planetaria, sin que esto signifique recesión o crecimiento negativo del PBI, sino una manera de independizarnos del economicismo por sí mismo para coexistir con la capacidad de carga ecosistémica del planeta (Giacomo et al., 2018; Demaria y Latouche, 2019). Esto significa que es necesario crear un espacio seguro y justo para la humanidad entre límites de dos tipos: (1) fundamentales, para proporcionar las necesidades sociales donde todas las situaciones no abastecidas serían consideradas una deficiencia; y (2) ecológicos, para controlar nuestro impacto en el mundo, donde todas las situaciones que los transgredan serían consideradas un sobrepasamiento. Vale mencionar que estas son las bases del modelo económico de la dona (véase figura 8), propuesto por Kate Raworth en el año 2017, que Ámsterdam plantea aplicar a partir del 2020.

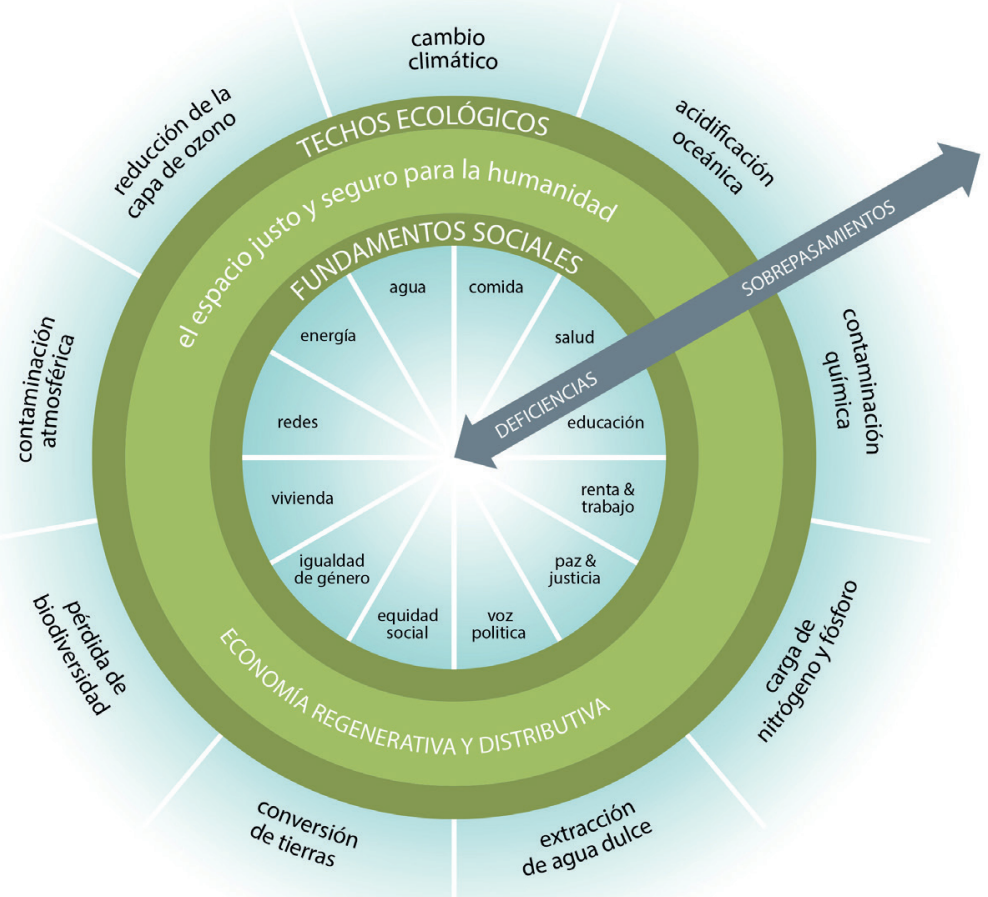

Figura 4.

La dona: una brújula del siglo XXI

Elaboración propia con base en Raworth (2017)

Estos límites, los fundamentales y ecológicos, son medidos por indicadores socioeconómicos (Raworth, 2017, pp. 239-243). Sin embargo, al aplicar este modelo en nuestro Sur Global, se deben añadir también nuevos límites de carácter ontoespistémicos. Por ejemplo, el derecho a la interculturalidad debería ser un límite fundamental, y esto significaría aceptar las formaciones 
ontológicas de nuestras cosmovisiones ancestrales, como el reconocimiento jurídico y político de la naturaleza. Por otro lado, la no extinción de estas mismas cosmovisiones debería ser un techo ecológico, pues son estos pueblos, que viven en relación con la naturaleza amenazada, los que luchan por su protección y muchas veces se oponen a los megaproyectos capitalistas de carácter dualista. Al optar por la durabilidad planetaria, es necesario dejar el crecimiento económico como objetivo social e ir en transición hacia un sistema civilizador justo y seguro para toda la humanidad. Después de todo, "lo que está en juego es 'territorios', pero en su amplia concepción material, epistémica, cultural y ontológica” (Escobar, 2014, p. 22; cursivas en el original).

\section{CONCLUSIONES Y RECOMENDACIONES}

Por un lado, la mayoría de estos límites, sean los propuestos por Raworth (2017) o los agregables para el Sur Global, se articulan con el territorio en sus distintas escalas. Por otro lado, regresando a la tabla 2, se demostró que los principales detonantes de los conflictos sociales en el Perú suceden en el ámbito territorial. Ambos comparten al territorio como su campo de acción y, por ende, se evidencia que pueden ser planificados y monitoreados desde el ordenamiento territorial. En concreto, dentro de los límites socioeconómicos, la agricultura, como medio de vida, está relacionada con los límites fundamentales de comida y renta/trabajo. Con este último también se puede relacionar la diversificación con trabajos resilientes independientes del extractivismo, como el ecoturismo y la agroecología. Asimismo, los proyectos priorizados de infraestructura sanitaria, comunicacional, vivienda o equipamientos que los centros poblados en influencia extractiva requieren se encuentran determinados por los límites fundamentales de agua, energía, redes, vivienda, educación y salud, respectivamente. Además, las áreas protegidas están relacionadas con los techos ecológicos de pérdida de biodiversidad, conversión de tierras y extracción de agua dulce (necesaria para el medio de vida agropecuario). Y, por último, todos los mencionados tienen que ver con los límites de carácter ontoespistémicos, ya que incluyen maneras distintas de percibir, ver y entender el mundo.

Es por esta razón que este artículo propone al ordenamiento territorial como una herramienta para la gestión de conflictos sociales, puesto que en palabras de De Echave et al. (2007):

No es posible resolver el conflicto minero, por lo tanto, sino ensanchando la discusión al problema mayor del ordenamiento del territorio: distribución de actividades económicas, planes de expansión urbana, sistemas de infraestructura vial, etc. No puede ser cabalmente enfrentado sino mediante la discusión, a nivel de un verdadero proyecto de país, donde el diseño territorial se convierta en el hilo conductor del debate económico y social. Un debate [...] donde la actividad minera encuentre su lugar (en el sentido metafórico, pero también literal de la palabra), junto con otras actividades (agricultura, turismo, industrias de transformación, etc.). Es solo de esta manera que las poblaciones directamente concernidas por los proyectos mineros podrán evaluar la pertinencia de la reconversión productiva de sus territorios [...] y las oportunidades de reorientación de 
sus vidas que esta reconversión implicaría. La actividad minera en el Perú tiene que ser parte de un proyecto colectivo, que cobije las peculiaridades territoriales y sociales del país y que brinde la oportunidad para cambios sociales deseados y no impuestos. (p. 15)

En este proceso es necesaria también la ecologización de la política normativista (Gudynas, 2014, pp. 3-5) creando nuevos dispositivos legales que permitan lo expuesto anteriormente. La urgencia mayor del Perú es establecer un organismo autónomo que fiscalice asuntos ambientales, pues actualmente el Ministerio de Energía y Minas, encargado de promover la inversión minera, es también el encargado de aprobar los estudios de impacto ambiental (De Echave, 2012, p. 79 ${ }^{22}$. Desde esta institucionalidad se impulsarían marcos legislativos como una ley del ordenamiento territorial y el reconocimiento a la autonomía territorial indígena para asegurar el reconocimiento verdadero de las consultas previas a los pueblos indígenas ${ }^{23}$. Además, la revisión al artículo $7^{24}$ de la Ley de Tierras, que permite la indemnización forzada a tierras comunitarias para proyectos mineros o de hidrocarburos (Ley 26570, 1996), o la aprobación del Proyecto de Ley 4369, que propone invertir el canon y regalías mineras para cerrar las brechas de infraestructura y pobreza en comunidades afectadas ("Ejecutivo prevé garantizar inversiones en comunidades", 2019, párr. 8). Estos son solo algunos de los apremios legislativos que marcarían un inicio para instaurar los límites fundamentales y ecológicos.

En conclusión, en entornos de extractivismo, no es posible separar la opresión a nuestros pueblos indígenas de la crisis civilizadora-ecológica mundial; ambas tienen un origen compartido en una visión cartesiana del mundo donde se evidencia cada vez más la necesidad de una transición hacia una sociedad de convivialidad (Illich, 1973). La introducción de distintas iniciativas interdisciplinarias en nuestro modelo, enmarcadas en discursos de transición como el decrecimiento, es la semilla de este proceso cuyo objetivo es un pluriverso ontoepistémico. El ordenamiento territorial, como una de estas iniciativas para entornos de extractivismo, nos permite reimaginar el territorio delimitando la actividad extractiva, los centros poblados y los territorios indígenas. La planificación otorga distintos horizontes temporales desde los cuales es posible avanzar hacia un pluriverso en el corto plazo gestionando los conflictos sociales al reconocer las cosmovisiones indígenas; en el mediano, consolidando una vida futura resiliente para evitar comunidades monoindustriales; y, en el largo plazo, coexistiendo dentro de los límites ecológicos de nuestro planeta.

22 Senace, órgano del Ministerio del Ambiente, solo se encarga de los megaproyectos que requieren estudio de impacto ambiental detallado. A la fecha (septiembre del 2020) esto equivale solamente a cuatro proyectos de extractivismo.

23 El artículo 15 de la Ley 29785, Ley del Derecho a la Consulta Previa a los Pueblos Indígenas u Originarios, otorga al Estado la decisión final sobre un proceso de consulta previa.

24 Modificado por la Ley 26570. 


\section{REFERENCIAS}

Angotti, T. (2013). Introduction Urban Latin America: Violence, Enclaves, and Struggles for Land. Latin American Perspectives, 40(2), 5-20. https:// journals.sagepub.com/doi/pdf/10.1177/0094582X12466832

Beteta, J. I. (25 de junio del 2019). El mal uso del canon minero en Perú / Entrevistado por BNamericas. BNamericas. https://www.bnamericas.com/es/entrevistas/ falta-foto-el-mal-uso-del-canon-minero-en-peru

Castellares, R., y Fouché, M. (2017). Determinantes de los conflictos sociales en zonas de producción minera [Documento de trabajo n. ${ }^{\circ}$ 2017-005, Banco Central de Reserva del Perú]. https://www.bcrp.gob.pe/docs/Publicaciones/ Documentos-de-Trabajo/2017/documento-de-trabajo-05-2017.pdf

Comisión Brundtland. (1987). Informe de la Comisión Mundial sobre el Medio Ambiente y el Desarrollo. Organización de las Naciones Unidas. http:// www.ecominga.uqam.ca/PDF/BIBLIOGRAPHIE/GUIDE_LECTURE_1/ CMMAD-Informe-Comision-Brundtland-sobre-Medio-Ambiente-Desarrollo. pdf

Correa Aste, N. (2011). Interculturalidad y politicas públicas: una agenda al 2016. CIES, Pontificia Universidad Católica del Perú; Consorcio de Investigación Económica y Social. http://cies.org.pe/sites/default/files/investigaciones/ interculturalidaddocumento.pdf

Damonte, G., Díez Hurtado, A., Fort, R., Pasco-Font, A., y Salas, G. (2003). Aprendiendo mientras se trabaja. En G. McMachon y F. Remy (Eds.), Grandes minas y la comunidad: efectos socioeconómicos y ambientales en América Latina, Canadá y España (pp. 145-202). Banco Mundial; Centro Internacional de Investigaciones para el Desarrollo; Alfaomega Colombiana.

De Echave, J. (2012). La minería peruana y los escenarios de transición. En A. Alayza y E. Gudynas (Eds.), Transiciones. Postextractivismo y alternativas al extractivismo en el Perú (pp. 59-86). CEPES.

De Echave, J., Diez, A., Huber, L., Revesz, B., Ricard, X., y Tanaka, M. (2009). Minería y conflicto social. Centro Bartolomé de las Casas; Centro de Investigación y Promoción del Campesinado; Consorcio de Investigación Económica y Social; Instituto de Estudios Peruanos. https://repositorio.iep.org.pe/ bitstream/IEP/639/2/huber_mineriayconflictosocial.pdf

De Echave, J., Tanaka, M., Huber, L., Revesz, B., Ricard, X., y Diez, A. (2007). Minería y conflicto social. Economía y sociedad, 65, 7-17.

Defensoría del Pueblo. (2020). Reporte de conflictos sociales $n .^{\circ} 198$. https://www. defensoria.gob.pe/wp-content/uploads/2020/09/Reporte-Mensual-deConflictos-Sociales-N\%C2\%B0-198-agosto-2020.pdf

Delbene-Lezama, L. (2015). Género, ecología y sustentabilidad. Documentos de Trabajo CLAES n. ${ }^{\circ}$ 73. http://ambiental.net/wp-content/uploads/2015/12/ DelbeneGeneroEcologiaDesarrollo.pdf 
Demaria, F., y Latouche, S. (2019). 37. Decrecimiento. En A. Acosta, F. Demaria, A. Escobar, A. Salleh y A. Kothari (Coords.), Pluriverso: un diccionario del posdesarrollo (pp. 204-207). Icaria Editorial.

Diego M. (1 de mayo del 2008). Monumento a la maca [Fotografía]. Flickr. https:/l www.flickr.com/photos/dmbpoe/2574909212

Domínguez, J. C. (2015). Megaproyectos fallidos en Latinoamérica: sociología histórica y política comparada. Instituto Mora.

El Comercio. (2 de septiembre de 2014). La SNMPE cuestiona el mal uso que se le ha dado al canon minero. El Comercio. https://elcomercio.pe/economia/peru/ snmpe-cuestiona-mal-le-dado-canon-minero-176408

Ejecutivo prevé garantizar inversiones en comunidades. (24 de mayo del 2019). Diario oficial El Peruano. https://www.elperuano.pe/noticia-ejecutivo-prevegarantizar-inversiones-comunidades-79827.aspx

Escobar, A. (2012). Más allá del desarrollo: postdesarrollo y transiciones hacia el pluriverso. Revista de Antropología Social, 21, 23-62. https://doi. org/10.5209/rev_RASO.2012.v21.40049

Escobar, A. (2014). Sentipensar con la tierra. Nuevas lecturas sobre desarrollo, territorio y diferencia. Universidad Autónoma Latinoamericana.

Escobar, A. (2016). Autonomía y diseño. La realización de lo comunal. Universidad del Cauca.

Feierabend, I., Feierabend, R., y Nesvold, B. (1969). Social Change and Political Violence: Cross-National Patterns. En H. D. Graham y T. R. Gurr (Dirs.), Violence in America: Historical and Comparative Perspectives (vol. 2, pp. 497-545). US Government Printing Office. https://play.google.com/books/ reader?id=qhHzWdpzSqYC\&hl=en\&pg=GBS.PA281

Feres, J. C., y Mancero, X. (2001). El método de las necesidades básicas insatisfechas (NBI) y sus aplicaciones en América Latina. Organización de las Naciones Unidas. https://www.mef.gob.pe/contenidos/pol_econ/documentos/Metodo _de_NBIs.pdf

Giacomo, D., Demaria, F., y Kallis, G. (2018). Decrecimiento. En D. Giacomo, F. Demaria y G. Kallis (Eds.), Decrecimiento: un vocabulario para una nueva era (pp. 36-59). Icaria Editorial; Fundación Heinrich Boell.

Gómez-Baggethun, E. (2019). Desarrollo sostenible. En A. Acosta, F. Demaria, A. Escobar, A. Salleh y A. Kothari (Eds.), Pluriverso: un diccionario del posdesarrollo (pp. 105-108). Icaria Editorial.

Gonzales Gavilano, A. (8 de diciembre del 2017). Minería, formas de urbanización y transformación del espacio en Huamachuco, La Libertad, Perú. Bulletin de l'Institut Français d'études Andines, 3(46), 509-527.

González Díaz, S. (2018). Reducciones. La concentración forzada de las poblaciones indígenas en el Virreinato del Perú. Historia, 51(1), 291-293. https://scielo.conicyt.cl/scielo.php?script=sci_arttext\&pid=S0717- 
71942018000100291\#: :text=Reducciones.,en\%20el\%20Virreinato\%20 del\%20Per\%C3\%BA.\&text=Una\%20reducci\%C3\%B3n $\% 20$ es $\% 20$ una\%20unidad,a\%20la\%20vida\%20en\%20polic\%C3\%ADa

Google Earth. (s. f.). [Ciudad de Cerro de Pasco]. Recuperado el 10 de septiembre del 2021 de https://earth.google.com/web/search/Cerro+De+Pasco/@10.67075929,-76.26482037,4299.6423155a,11142.46215929d,35y,0h ,44.998222t,0r/data=CnkaTxJJCiUweDkxMDg2ZDVhNWVIOGM3YTk 6MHg5MDMONTAwZmE1ZmJjM2FkGUz-J3_3WiXAIRLuOsC4EFPAK g5DZXJybyBEZSBQYXNjbxgBIAEiJgokCcHwOFZWbjNAEb3wOFZW bjPAGXRqkqaTDj9AIQkh-zvMW1HA

Gudynas, E. (2009). Desarrollo sostenible: posturas contemporáneas y desafíos en la construcción del espacio urbano. Vivienda Popular, 18, 12-19.

Gudynas, E. (2013). Extracciones, extractivismos y extrahecciones. Un marco conceptual sobre la apropiación de recursos naturales. Observatorio del Desarrollo, 18, 1-18. http://ambiental.net/wp-content/uploads/2015/12/ GudynasApropiacionExtractivismoExtraheccionesOdeD2013.pdf

Gudynas, E. (2014). Ecologías políticas. Ideas preliminares sobre concepciones, tendencias, renovaciones y opciones latinoamericanas. Documentos de Trabajo CLAES, n. ${ }^{\circ}$ 72. http://ecologiasocial.com/wp-content/uploads /2016/09/EcologiaPoliticaDefinicionesTendenciasGudynasDT2014.pdf

Gurr, T. R. (1970). Why Men Rebel. Princeton University Press.

Harvey, D. (2003). El nuevo imperialismo. Akal.

Illich, I. (1973). Tools for Conviviality. Harper \& Row.

Instituto Nacional de Estadística e Informática. (septiembre de 1995). 3.1.2 El crecimiento de las ciudades. https://www.inei.gob.pe/media/MenuRecursivo /publicaciones_digitales/Est/Lib0018/cap31002.htm

Instituto Nacional de Estadística e Informática. (2008). Perfil sociodemográfico del Perú. https://www.inei.gob.pe/media/MenuRecursivo/publicaciones_digitales/ Est/Lib1136/libro.pdf

Instituto Nacional de Estadística e Informática. (2018). Perú: Perfil sociodemográfico. Informe nacional. https://www.inei.gob.pe/media/MenuRecursivo/ publicaciones_digitales/Est/Lib1539/libro.pdf

Johnson, C. (1966). Revolutionary Change. Little, Brown and Company.

Kunanayagam, R., McMahon, G., Sheldon, C., Strongman, J., y Weber-Fahr, M. (2001). Mining and Poverty Reduction. Banco Mundial. https://pdfs. semanticscholar.org/dc16/d51f66f94d11d41ddc2f8b8855d2f94e5778.pdf

Law, J. (2011). What's Wrong with a One-World. Heterogeneities. http://www. heterogeneities.net/publications/Law2011WhatsWrongWithAOne WorldWorld.pdf

Ley 26570 de 1996 . Sustituye artículo de la Ley $n .^{\circ} 26505$ referido a la utilización de tierras para el ejercicio de actividades mineras 0 de hidrocarburos. 
4 de enero de 1996. http://intranet2.minem.gob.pe/web/archivos/dgm/ legislacion/LEY\%20N_26570.pdf

Ley 30848 del 2018. Ley que modifica la Ley 27506 , Ley de Canon, a fin de promover el financiamiento de programas de vivienda social. 28 de agosto del 2018. Normas Legales n. ${ }^{\circ} 14655$, diario oficial El Peruano. https://busquedas. elperuano.pe/normaslegales/ley-que-modifica-la-ley-27506-ley-de-canona-fin-de-promov-ley-n-30848-1693569-1/

Loayza, N., y Rigolini, J. (2015). The Local Impact of Mining on Poverty and Inequality: Evidence from the Commodity Boom in Peru [Working paper n. ${ }^{\circ} 33$ ]. http:/l perueconomics.org/wp-content/uploads/2014/01/WP-33.pdf

López García, D., y López López, J. A. (2003). Con la comida no se juega: alternativas autogestionarias a la globalización capitalista desde la agroecología y el consumo. Traficantes de Sueños.

Lorenzo Cadarso, P. L. (2001). Principales teorías sobre el conflicto social. Norba: Revista de Historia, 15, 237-254.

Macrocontenidos. (14 de abril del 2017). Estadio 25 de Noviembre 2017 [Archivo de video]. YouTube. https://www.youtube.com/watch?v=ulWpcpP7018\&t=7s

Meadows, D. H., Meadows, D. L., y Randers, J. (1994). Más allá de los límites del crecimiento. El País; Aguilar.

Ministerio de Vivienda, Construcción y Saneamiento. (2008). Plan de Desarrollo Urbano de la Ciudad de Cerro de Pasco 2006-2016. VolumenA: Diagnóstico. https://eudora.vivienda.gob.pe/observatorio/PDU_MUNICIPALIDADES/ CERRODEPASCO/PDU_CERRODEPASCO_DIAGNOSTICO.pdf

Montero Peña, J. M., y Salazar Pérez, Y. (2012). La reinserción laboral tras el cierre de minas: una vía para lograr el desarrollo sustentable en la minería. Minería y Geología, 27(4), 64-87. https://pdfs.semanticscholar. org/9957/6219414d37e8bd4e7c55e2736c6f8435c49f.pdf

Olmedo Neri, R. (2019). La renta de tierra en las zonas rurales de México: un estudio de caso sobre los efectos de la nueva ruralidad. Espacio Abierto. Cuaderno Venezolano de Sociología, 28(2), 153-169.

Parra Romero, A., y Cadena Díaz, Z. (2010). El medio ambiente desde las relaciones de ciencia, tecnología y sociedad: un panorama general. Revista CS, 6 , 331-359.

Perú21. (23 de junio del 2013). Fotos: Los monumentos a la ridiculez. Perú21. https:// peru21.pe/lima/fotos-monumentos-ridiculez-112425-noticia/?foto=5

Pineda, C. E. (2018). El despliegue del capital sobre la naturaleza. En H. Cuevas Valenzuela, D. Julián Véjar y J. Rojas Hernández (Eds.), América Latina: expansión capitalista, conflictos sociales y ecológicos (pp. 125-146). RIL Editores; Universidad de Concepción.

Porto Gonçalves, C. W. (2001). Geo-grafías: movimientos sociales, nuevas territorialidades y sustentabilidad. Siglo XXI Editores. 
Quispe, A. (21 de diciembre del 2013). Cosmovisión andina. Takiruna. https://takiruna. com/2013/12/21/cosmovision-andina/

Raworth, K. (2017). Doughnut Economics: Seven Ways to Think Like a 21st-Century Economist. Random House Business Books.

Rodríguez, J. A. (9 de septiembre del 2012). Mirador de la Biodiversidad, en el centro de Puerto Maldonado [Fotografía]. Travesías \& Momentos. http:/l travesiasymomentos.blogspot.com/

Ross, M. (1999). The Political Economy of the Resource Curse. World Politics, 51(2), 297-332.

Rota Verbum. (18 de enero del 2018). Raúl Zibechi - Crisis: ¿Económica o civilizatoria? [Archivo de video]. YouTube. https://www.youtube.com/ watch?time_continue $=1 \& v=z q X A u D Z y R F I \&$ feature $=e m b \_t i t l e$

Sossa Rojas, A. (2010). La alienación en Marx: el cuerpo como dimensión de utilidad. Revista de Ciencias Sociales, 25, 37-55. https://www.redalyc.org/ pdf/708/70817741003.pdf

Svampa, M. (2019). Las fronteras del neoextractivismo en América Latina. Calas. http://calas.lat/sites/default/files/svampa_neoextractivismo.pdf

Thodes Miranda, E. (2016). Segregación socioespacial en ciudades mineras: el caso de Antofagasta, Chile. Notas de Población, 43(102), 203-227.

Truman, H. S. (20 de enero de 1949). Inaugural Address. https://www.bartleby. com/124/pres53.html

Van Houtryve, T. (2 de diciembre del 2015). El centro histórico del Cerro de Pasco ha sido consumido por una mina de más de media milla de profundidad [Fotografía]. National Geographic. https://www.nationalgeographic.es/ arqueologia/en-los-andes-una-mina-esta-punto-de-tragarse-una-ciudadde-400-anos-de-antiguedad

Vittor, L. (6 de julio del 2007). Perú: Cerro de Pasco y la expansión minera, un conflicto infinito. BiodiversidadLa. http://www.biodiversidadla.org/Noticias/ Peru_Cerro_de_Pasco_y_la_expansion_minera_un_conflicto_infinito 